\title{
Requisitos de estabilidad y de durabilidad de los áridos del hormigón
}

Informe completo sobre la conferencia pronunciada en el IETcc, el día 16 de octubre de 1975, por M. LEVITT, Ph. D.

Agradezco a los Profesores Arredondo y Calleja, su amable invitación para visitar el IETcc y poder dirigirme a todos Vds. Este es mi tercer viaje a. España este año y es un placer encontrarse en un país tan civilizado.

Cuando se habla acerca de durabilidad, se debe de definir el término, en el sentido de indicar si se trata de los áridos o del hormigón. Yo defino la palabra como la capacidad de un material para mantener sus propiedades calculadas durante un período de vida útil previsto. En España, muchas veces la palabra durabilidad se emplea para la resistencia a los sulfatos, pero éste es sólo un aspecto de la durabilidad. Hay otros muchos riesgos, por ejemplo, los producidos por el mar, por las heladas, por el fuego, en construcciones industriales y agropecuarias, etc. La durabilidad de un hormigón es función de la dosificación, de la compactación y del curado, y el control simultáneo de estos parámetros es muy importante. Se corre mucho riesgo si no se controla uno de estos parámetros, pues también se puede olvidar cualquiera de los otros dos. El tema que se va a desarrollar se refiere a un aspecto de la confección del hormigón, cual es la selección del árido.

La estabilidad y la resistencia son propiedades parcialmente relacionadas con la durabilidad, por lo tanto vale la pena dedicar algún tiempo a tratar de ellas.

En el Reino Unido se utiliza el Valor de Rotura (VR) de la Norma Británica 812, el cual consiste en aplicar una carga de $40 \mathrm{t}$ sobre una muestra de $3 \mathrm{~kg}$ en un molde de $15,4 \mathrm{~mm}$ de diámetro. El valor VR se registra como el porcentaje que pasa a través de un tamiz de $2,4 \mathrm{~mm}$ de luz de malla. Es inversamente proporcional a la resistencia a compresión (RC); por ejemplo:

$$
\begin{aligned}
& \text { Cuarcita, } \mathrm{RC}=135-200 \mathrm{~N} / \mathrm{mm}^{2} \ldots \ldots \ldots \ldots \ldots \ldots \\
& \text { Arenisca, } \mathrm{RC}=33-52 \mathrm{~N} / \mathrm{mm}^{2} \ldots \ldots \ldots \ldots \ldots \ldots \text { VR }=36,-\%
\end{aligned}
$$

La fórmula aproximada es $\mathrm{RC}=300-6,73 \mathrm{VR}$.

Para una resistencia razonable de un árido se sugiere un límite de un $30 \%$ (máximo).

El valor de diez por ciento de finos es la carga en toneladas requerida para dar un VR del $10 \%$. Es obvio que hay una estrecha relación con el ensayo del VR y se sugiere un límite de 10 t para el valor del $10 \%$. 
En la Norma Británica se incluye el ensayo del Valor del Impacto (VI). Este comprende el porcentaje de polvo producido por choques repetidos y está evidentemente relacionado con el valor de rotura tal como muestra la tabla siguiente:

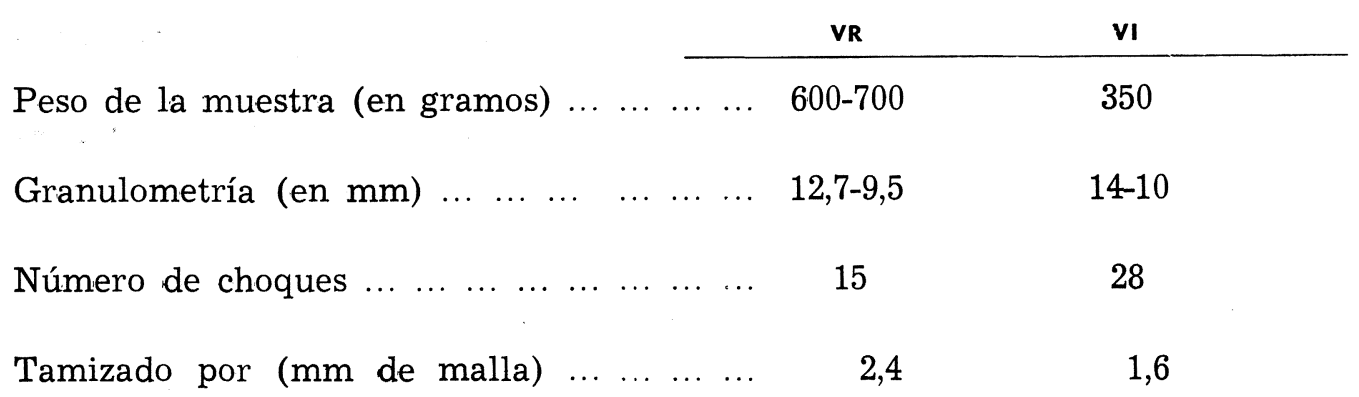

Se sugiere un límite de un $25 \%$ para el valor del impacto.

Nota.-Este ensayo guarda, también, una gran similitud con el Ensayo de abrasión de Los Angeles.

En rocas sedimentarias y metamórficas con estructura laminar como por ejemplo, pizarras, esquistos y areniscas, puede tener lugar una separación en forma de hojas o de láminas. Muy a menudo, el agua queda atrapada entre estas capas y al calentar a $700^{\circ} \mathrm{C}$ durante 30 minutos se elimina este agua, produciéndose una expansión con la consiguiente disrupción. El ensayo no debe de hacerse con rocas cuarcíticas, debido al cambio de fase a los $573^{\circ} \mathrm{C}$; también deben excluirse las rocas carbonatadas, debido a su descomposición parcial. De unas 100 muestras de áridos ensayadas, no deben fallar más del $4 \%$. La mayoría de los áridos dan un porcentaje de 0 a 0,5\%; un par de esquistos ensayados dieron porcentajes de rotura del 11 al $14 \%$. Este ensayo se puede utilizar para tener una orientación acerca de la resistencia al fuego del árido.

Una vez discutidos estos requisitos generales se va a hablar ahora acerca de los ensayos acelerados de laboratorio, asimilables a los procesos naturales de envejecimiento.

El comportamiento térmico a temperaturas elevadas crecientes se consideró una idea adecuada y los valores del impacto se pueden determinar en distintas muestras calentadas a 100,400 y $700^{\circ} \mathrm{C}$, siendo enfriadas, cada una de ellas, a la temperatura ambiente, antes de los ensayos. Los valores a estas respectivas temperaturas son $\mathrm{I}_{1}, \mathrm{I}_{2}$ e $\mathrm{I}_{3}$.

El criterio de valor $\left(I_{3}\right.$ situado por encima de la recta $\left.I_{1} I_{2}\right)$ es:

$$
100 \times \frac{\mathrm{I}_{1}+\mathrm{I}_{3}-2 \mathrm{I}_{2}}{\mathrm{I}_{1}}
$$

El ensayo mide los efectos de los productos de alteración y no de la expansión térmica por sí misma. Un límite del orden del $40 \%$ es aproximadamente el correcto, pero se requieren más datos.

El ensayo con sosa cáustica $(\mathrm{NaOH})$ detecta la presencia de caolín en la reacción feldespática en rocas arcillosas tales como la arcilla esquistosa, la pizarra y el esquisto. Una disolución de $\mathrm{NaOH}$ al $5 \%$ hace flocular al caolín y la velocidad de sedimentación, puede utilizarse como medida de la cantidad de material reactivo. Los áridos gruesos se trituran hasta hacerlos pasar por un tamiz de 60 micras y una muestra representativa de 
5 gramos se coloca en una probeta de $100 \mathrm{ml}$, se añaden $100 \mathrm{ml}$ de $\mathrm{NaOH}$ al $5 \%$ y se agita la probeta durante 15 segundos. Las lecturas del volumen de los productos floculados se toman a los 10 minutos y luego a las 2 horas; pero la lectura echa a los 10 minutos es más sensible. Se considera un límite del orden de 15-25 ml. Los áridos que se comportan bien dan a los 10 minutos lecturas de $5-13 \mathrm{ml}$; los áridos pobres dan lecturas que alcanzan hasta los $36 \mathrm{ml}$.

El ensayo hecho con peróxido de hidrógeno $\left(\mathrm{H}_{2} \mathrm{O}_{2}\right)$ orienta acerca de la aptitud a la expansión de rocas con arcilla en su estructura, tales como la montmorillonita, la vermiculita y la hidrobiotita, las cuales pueden expandirse debido a la fijación de agua. Una muestra de $1 \mathrm{~kg}$ constituida por tamaños comprendidos entre 10-20 $\mathrm{mm}$ se coloca en un vaso de precipitado de 2 litros y se añade 1 litro de peróxido de hidrógeno de 110 volúmenes $(33 \%)$. Se ajusta el $\mathrm{pH}$ a 2,5 (con ácido sulfúrico o con sosa cáustica) y la disolución, con el vaso de precipitados cubierto, se lleva hasta ebulición, y después se mantiene en una estufa a $110^{\circ} \mathrm{C}$ durante 18 horas. Luego se enfría y se lava sobre un tamiz de $10 \mathrm{~mm}$ de luz de malla, se calienta en estufa a $105^{\circ} \mathrm{C}$ durante 12 horas o más; después se tamiza en seco con otro tamiz de $8 \mathrm{~mm}$ de luz de malla. La relación entre el árido pequeño que pasa por el tamiz de $8 \mathrm{~mm}$ y el total, es una medida de la arcilla reactiva. Cualquier resultado superior al $2 \%$ supone un riesgo, y por encima del $5 \%$ ciertamente causa daño al hormigón.

Las zonas de árido bueno y malo tienen un amplio espacio de solapamiento y no es fácil fijar un límite a un nivel definido.

Otra forma de desintegración natural es la acción del hielo y del deshielo y, si bien en España no existe ese riesgo en general, no obstante se debe de tener en cuenta, ya que los áridos y los hormigones en regiones montañosas pueden estar sometidos a dicha acción.

El primer ensayo sobre áridos desarrollado por Tourenq, consistió en el Valor de Impacto VI, sobre muestras separadas antes y después de ser sometidas a 25 ciclos, cada uno de ellos de 12 horas, a temperaturas comprendidas entre $-25^{\circ} \mathrm{C}$ y $+25^{\circ} \mathrm{C}$. Los resultados muestran siempre una amplia diversidad, y de 5 áridos nocivos "propensos al hielo", sólo uno se manifestó como no apto según este ensayo. El otro ensayo desarrollado por mí mismo, consiste en tomar 100 trozos representativos del árido, empaparlos en agua a presión atmosférica normal, someterlos a 5 ciclos de hielo-deshielo, cada uno de 24 horas de duración, entre $-20^{\circ} \mathrm{C}$ y $+20^{\circ} \mathrm{C}$, y contar después el número de trozos que quedan enteros. Generalmente se termina con 100 piezas mas unas cuantas escamas o laminillas pequeñas; de 5 áridos nocivos ensayados se encontraron 80-90 piezas que quedaron enteras. Se sugiere un límite de 100 piezas mas unas cuantas laminillas o escamas, es decir, que no haya trozos deteriorados, para definir un árido como resistente a las heladas.

Un ensayo similar es el de estabilidad, consistente en hacer crecer cristales de sulfato de forma que se produzca un aumento de volumen semejante al producido en la formación del hielo. Se puede utilizar el método de ensayo ASTM, C 88-73 con un límite del $18 \%$ después de 5 ciclos, tal y como se especifica en el ASTM, C 33-71. Dos de los áridos nocivos se comportaron bien en este ensayo, y no existe correlación alguna entre el resultado del mismo y el ataque producido por la helada. El último trabajo del Concrete Durability Committee (CDC) de la RILEM arguye que esto no es sorprendente, dado que el daño producido por el hielo en materiales permeables, es debido principalmente a la ineptitud de la estructura capilar para acomodar agua superenfriada.

El ensayo de expansión térmica mide la diferencia entre el comportamiento de la matriz o pasta cementicia y el del árido pétreo. Si esta diferencia es grande se puede producir desunión entre ambos. Midiendo el comportamiento de muestras húmedas entre $-20^{\circ} \mathrm{C}$ 
y $+20^{\circ} \mathrm{C}$ se encuentra, de acuerdo con Callan, que se pueden tolerar diferencias menores que $5,4 \times 10^{-6} /{ }^{\circ} \mathrm{C}$. La expansión que se produce por humidificación es pequeña y las diferencias de absorciones de agua en rocas naturales y en prismas de mortero no se consideraron importantes. Las expansiones reales de las rocas se sitúan en la zona de 5 a $6 \times 10^{-6} /{ }^{\circ} \mathrm{C}$, mientras que las del mortero se sitúan en la zona de 10 a $12 \times 10^{-6} /{ }^{\circ} \mathrm{C}$. En efecto, es posible escoger una roca con una elevada expansión térmica relativa, que dé lugar a la desunión entre la pasta y el árido. Las absorciones de agua de la piedra natural son de 0,1 a $2 \%$ y las de los morteros de 6,2 a 7,3, valores que no pueden explicar las anteriores diferencias.

Ahora llegamos a aspectos de la durabilidad intrínseca del hormigón, es decir, al modo de comportarse los áridos cuando están rodeados de una matriz cementante, esto es, de pasta de cemento.

El primer riesgo bien conocido es la interacción álcalis silicatos, por la que ciertos áridos tales como el ópalo, la calcedonia y la tridimita reaccionan con los álcalis del cemento provocando expansión. La Norma ASTM C 227-71, mide la expansión de prismas de mortero, y el Ensayo C 289-71 mide la cantidad de materiales reactivos en el árido. El primer ensayo es el más significativo y de una expansión superior a $0,05 \%$ en 3 meses, se dice que indica riesgo. Sin embargo, este riesgo se puede eliminar utilizando un cemento con bajo contenido en álcalis (por ejemplo de 0,6\% como máximo de $\mathrm{R}_{2} \mathrm{O}$ ), con lo cual se ha comprobado que los áridos dudosos se comportan bien.

La reacción álcalis-carbonato es también expansiva y se produce cuando grandes cristales de dolomita están diseminados dentro de una matriz de calcita/arcilla finamente granulada. A medida que la arcilla absorbe lentamente agua, se produce una reacción conocida como desdolomitización:

$$
\mathrm{CaMg}\left(\mathrm{CO}_{3}\right)_{2}+2 \mathrm{NaOH}=\mathrm{Mg}(\mathrm{OH})_{2}+\mathrm{CaCO}_{3}+\mathrm{Na}_{2} \mathrm{CO}_{3}
$$

Se puede emplear el Ensayo ASTM C 586-69 consistente en sumergir las muestras en disolución de $\mathrm{NaOH}$, pero así no se encontrarían áridos nocivos en la Europa Nórdica; sin embargo, el problema podría surgir en la parte Oriental de España. La bibliografía indica que una expansión mayor de $0,1 \%$ es indicio de dificultades pero el ensayo debería extenderse gradualmente hasta muestras representativas de hormigón, de tamaño natural.

El cambio potencial de volumen se puede producir a causa de algunas combinaciones del árido y del cemento que dan lugar a una expansión peligrosa. El Ensayo ASTM, C 33-71, se realiza manteniendo los prismas a una humedad relativa de $60 \%$ y a $25^{\circ} \mathrm{C}$ durante un año, y cualquier expansión por encima del $0,2 \%$ se considera peligrosa. Como puede verse, el ensayo es de larga duración y el comportamiento estimado resulta de una combinación de los coeficentes de expansión térmica, de la unión del árido a la pasta y de la elasticidad del árido grueso. No se podría encontrar tal combinación en el Reino Unido.

Son bien conocidas las impurezas de piritas y pirrotita cuyas reacciones de oxidación dan lugar a manchas. La pirrotita está también sometida a la reacción de hidratación antes que a la de oxidación, y la expansión subsiguiente puede causar daños. Es decisivamente importante la selección de la cantera del árido. Un ensayo sencillo consiste en añadir ácido clorhídrico diluído al árido y detectar el olor del ácido sulfhídrico $\left(\mathrm{H}_{2} \mathrm{~S}\right)$.

Algunos áridos sintéticos con estructura vítrea pueden estar también sujetos a la reacción expansiva alcalis-silicatos. Los áridos ligeros al ser elásticos, posiblemente absorben esta expansión sin detrimento, pero algunos materiales tales como la escoria vítrea podrían causar dificultades. 
Veamos algo más acerca de la dosificación, de la compactación y del curado. La dosificación está controlada por dos requisitos básicos: la trabajabilidad necesaria y las propiedades (por ejemplo, la resistencia) deseadas. La compactación total es una exigencia general para la mayoría de los hormigones, pero los hormigones parcialmente compactados pueden ser durables si la corrosión del acero de las armaduras no supone ningún riesgo. La gran capilaridad de la estructura de los poros y huecos en tales hormigones pueden tolerar fácilmente las fuerzas expansivas ocasionadas por la mayor parte de los riesgos que dan lugar a ellas. El curado es más importante de lo que se cree, debido a que la mayor parte de los riesgos de la durabilidad empiezan en la superficie del hormigón. Como se ha dicho anteriormente, si no se controla cualquiera de estos aspectos, el control de los otros dos resulta inútil en muchas circunstancias.

Consideremos ahora las aplicaciones especiales del hormigón. En general, las carreteras de España no están sometidas a las heladas ni a los productos químicos de deshielo, pero los agentes inclusores de aire empleados en Europa pueden ser útiles para facilitar la trabajabilidad del hormigón fresco y para mejorar la impermeabilidad del hormigón endurecido. El deterioro por desgaste o atricción es un problema en cualquier parte y los áridos calizos se pueden desgastar o pulir fácilmente. Los áridos gruesos de caliza dura, no desgastable, son buenos como material grueso para hormigones de carreteras, pero el árido fino deberá ser siempre silíceo. Los hormigones para centrales nucleares necesitan ser de gran densidad y proporcionar estructuras no agrietadas o fisuradas, a fin de que protejan evitando fugas. Los perdigones o granalla de hierro, la barita, la magnetita, etc., son áridos de gran densidad, de los cuales se dispone y son aptos, pero hay que cuidar el curado para evitar las grietas. Los recipientes a presión constituyen otro campo que exige hormigones estructurales de la más alta calidad.

Las construcciones marítimas, industriales y agropecuarias ofrecen un amplio campo de ataque químico y es importante prestar atención extremada a la dosificación, a la compactación (en grado extremo) y al curado. En algunos casos el hormigón, independientemente de lo bien que se haya proyectado, no puede resistir al peligro, y ante tal riesgo es necesario un tratamiento protector. En construcciones agropecuarias es mejor evitar el empleo de áridos carbonatados, ya que los ácidos orgánicos los atacan preferentemente. También las estructuras que retienen agua, necesitan, de igual modo, una especial atención y el diseño de las juntas (las de dilatación y las de hormigonado) es asímismo importante. Como disgresión interesante, una o dos compañías del Reino Unido sostienen que es mejor no prestar atención a nada en la construcción de tales estructuras (permitiendo que se formen grietas en ellas) y poner después en su interior un recubrimiento impermeable. Los barcos de hormigón están cada vez más en boga y la mayoría de ellos consisten en estructuras fuertemente armadas y con morteros ricos en cemento. Ponen de manifiesto que la armadura de acero del hormigón, sin recubrimiento, puede experimentar corrosión superficial, pero nunca produce descascarillado, resquebrajamiento o agrietamiento. Generalmente, la superficie de la armadura se pinta después para detener la formación de herrumbre superficial y conseguir un efecto decorativo.

Para concluir, unas pocas palabras acerca del trabajo internacional del Comité de Durabilidad del Hormigón de la RILEM. Este ha existido durante 11 años y en los cuatro últimos, ha empezado a producir algún trabajo útil. El Código de Buena Práctica para Hormigón Durable se llevó a cabo como guía general acerca de los factores más importantes, pero se elaboró bajo el mínimo denominador común aceptable por todos los países y ocurrió que ni siquiera así resultó serlo. Ha sido publicado solamente en un país, pero se pueden obtener copias del informe.

Los trabajos sobre la resistencia química del hormigón se han llevado a cabo en estrecha relación con la investigación realizada por el CEMBUREAU, pero nadie está satisfecho 
con los ensayos normalizados para el cemento, cuando se aplican al hormigón. Se han preparado valiosos datos sobre resultados de la investigación, así como puestas a punto de los logros alcanzados en la misma.

Además de los informes de Bélgica y del Reino Unido, ha sido preparado un excelente trabajo por vuestro propio Profesor Calleja.

El ensayo de hielo-deshielo, con o sin productos químicos descongelantes, ha sido redactado y publicado en la revista Materials and Structures como Tentativa de Norma; en breve será publicado como Recomendación. Un trabajo adicional sobre el Método de Saturación Crítica, también será publicado en breve para su comentario internacional.

La RILEM no es partidaria de que los Comités se mantengan durante mucho tiempo y nuestra reunión que tuvo lugar aquí en Madrid el último mes de abril (1975) fue el último acontecimiento del CDC-4. Se propuso al Comite Permanente de la RILEM la idea de continuar la investigación y en su reunión del pasado mes en Edimburgo se tomó la decisión de crear un nuevo Comité que se ocupe de los "Métodos de Ensayo de la Resistencia Química del Hormigón". Espero que España con sus múltiples intereses en este tema, esté representada en el Comité de nueva creación. 